\title{
474858 - DIASTOLIC DYSFUNCTION AND POSTOPERATIVE ATRIAL FIBRILLATION IN PATIENTS UNDERGOING CORONARY REVASCULARIZATION SURGERY
}

\author{
Massimiliano Meineri, MD, Ronit Lavi, MD, Jo Carroll, RN, Jacek Karski, MD, \\ FRCPC, George Djaiani, MD \\ Department of Anesthesia and Pain Management, Toronto General Hospital, \\ Toronto, ON, Canada
}

Introduction: Atrial fibrillation (AF) occurs in 20-30\% of patients undergoing coronary revasularization surgery and is associated with significant perioperative morbidity and mortality.[1-2] During coronary artery bypass graft surgery (CABG), left ventricular (LV) diastolic dysfunction (DD) is a frequent occurrence.[3] The echocardiographic preditors of perioperative DD are not well defined. This study investigates a relationship between the perioperative DD and a new onset of AF.

Methods: Following REB approval, an informed consent was obtained from 72 patients undergoing elective CABG surgery. A complete transesophageal echocardiographic examination (TEE) was performed after induction of anesthesia and after surgical revacularization. The following measures were recorded in all patients: mitral valve inflow (e and a wave velocities, a/e ratio, deceleration time, a duration), pulmonary venous flow (s and d wave velocities, s/d ratio, duration of atrial reversal), mitral annular tissue doppler velocities (a' and e' wave velocities, e'/a' ratio). DD was assesses off-line by two independent investigators. DD was classified in three patterns (impaired relaxation, pseudo-normal and restrictive) according to the previously published guidelines.[4] The primary outcome was to assess if the presence of DD is associated with increased risk of postoperative AF.

Results: 18 of 72 patients (25\%) developed post-operative AF. Age, gender, co-existing diseases, number of grafts and duration of cardiopulmonary bypass were similar in the patients with and without DD. DD at baseline was not associated with increased risk of postoperative AF $(\mathrm{p}=0.20)$. Worsening of DD after revascularization was associated with a higher incidence of postoperative AF ( $p=0.035)$ (Fig.1).

Discussion: Worsening of DD after coronary revascularization should be considered as a risk factor for new onset AF after CABG surgery.

References: [1] J Am Coll Cardiol 2001;37:371-380, [2] J Am Coll Cardiol 1998;32: 695-703, [3] J Thorac Cardiovasc Surg 1997;114:254-60,[4] J of Am Soc Echocardiogr 1996;9:736-760. 
Fig.1 Diastolic function and new onset of postoperative atrial fibrillation
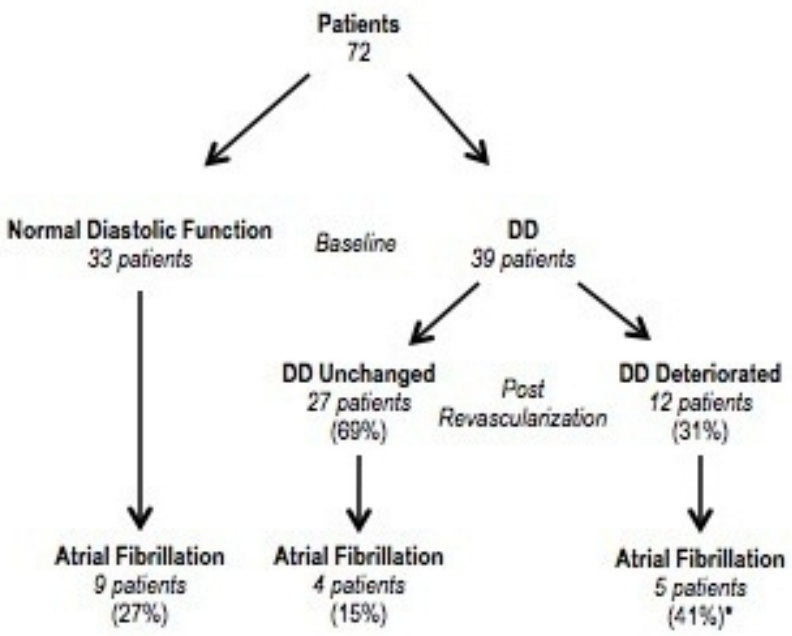

DD: Diastolic Dysfunction; * $p=0.035$ 